\title{
Hand Gesture Recognition for Real Time Human Machine Interaction System
}

\author{
Poonam Sonwalkar, Tanuja Sakhare, Ashwini Patil, Sonal Kale. \\ Nutan Maharashtra Institute of Engg and Technology, Talegaon Dabhade, Pune 410507. \\ Computer Department. \\ Guide by: Prof. Bhavana S. Pansare
}

\begin{abstract}
Real Time Human-machine Interaction system using hand gesture Recognition to handle the mouse event, media player, image viewer .Users have to repeat same mouse and keyboard actions, inducing waste of time. Gestures have long been considered as an interaction technique that can potentially deliver more natural. A fast gesture recognition scheme is proposed to be an interface for the human-machine interaction (HMI) of systems. The system presents some lowcomplexity algorithms and gestures to reduce the gesture recognition complexity and be more suitable for controlling real-time computer systems. In this paper we use the webcam for capturing the image. After capturing the image it converts into the binary image. A gesture is a specific combination of hand position.
\end{abstract}

Keywords - Gesture Recognition, Human Machine Interaction System, Webcam.

\section{INTRODUCTION}

The computers understand human language and develop a user friendly human computer interfaces (HCI). Gestures are the non-verbally exchanged information. The computer programmers have been incredibly successful in easing the communication between computers and human. The project aims to determine human gestures by creating an HCI. An overview of gesture recognition system is given to gain knowledge. A gesture is categorized into two distinctive categories: dynamic and static. The system uses Dynamic gesture because these are real time system. There are many challenges associated with the accuracy and usefulness of gesture recognition software.The main challenge is generating some noise in capturig image. The variety of implementations for image-based gesture recognition may also cause issue for the technology to general usage.

\section{EXISTING SYSTEM}

Previous system captures Black and White images and those gestures are predefined. It scans the whole body as input. It used hand gloves for gesturing. For controlling the games and smart phones it uses static gestures only. In previous system it have a problem that similar gestures might have different orientation histrograms and also different gestures have similar orientation of histrograms.

\section{PROPOSED SYSTEM}

The entire system captures the real-time image which is color image as input .The system uses serialized database for storing gestures. Well Accuracy and efficiency. Our project is that we are focusing on interaction between human and computer machine. The proposed scheme can be used to develop a realtime gesture recognition system. The first option is to eliminate most background regions to remain the hand region when the background of implemented environment is less complex. The second option is to detect hand regions by color or brightness. The hand region detection is a very important procedure for identifying the shape and area of each hand.. Our system can work well in real time service system.

\section{RELATED WORK}

The most gesture recognition methods usually contain three major stages. The first stage is the object detection. Many environment and image problems are needed to solve at this stage to ensure that the hand contours or regions can be extracted precisely to enhance the recognition accuracy. Common image problems contain unstable brightness, noise, poor resolution and contrast. The second stage is object recognition. The detected hand objects are recognized to identify the gestures. At this stage, differentiated features and effective classifiers selection are a major issue in most researches. The Third stage is instructs or behaviour of analysis. In this paper, motion-based dynamic gesture recognition system for interaction between human and computer system is proposed.

\section{SYSTEM ARCHITECTURE}

Fig. 1 shows the architecture of system:

1) Step 1-in system architecture first it captures image from webcam call as video capturing .

2) Step2- in second step our captured image is $2 \mathrm{D}$ it stored in 2D matrix from. 
3)step3- After storing image Apply croppin because neglecting background region, and only get image part.

4)Step4- After that apply scaling for zoom out or zoom in option.

5)Step5-After template generated like any symbol which is stored into the database like A,S,L call as template generated.

6)Step6- After template generation template matching occurred match symbol with database.

7)Step7- template fetching occurred nothing but calling from database for particular work.

Ones the templates are fetched then system performs different actions like mouse handling, Image viewer, Media player.

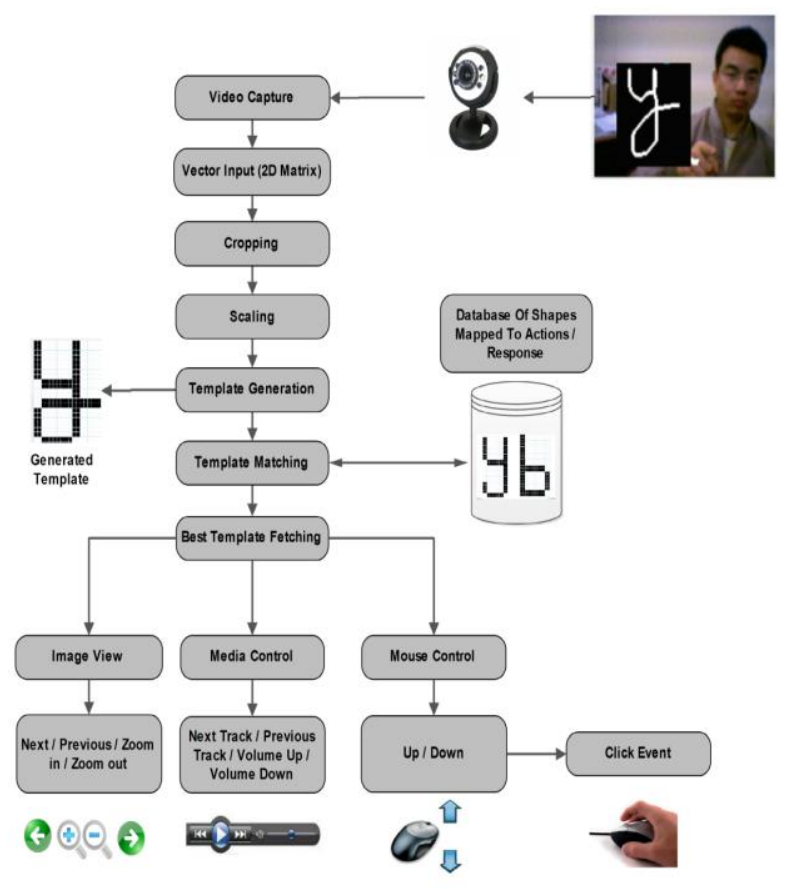

FIG. 1 SySTEM ARCHITECTURE

\section{ALGORITHM}

\section{RGB Color Model}

The RGB color model is an additive color model in which red, green, and blue color is added together in various ways to reproduce a broad array of colors.

In computing, the component values are often stored as integer numbers in the range 0 to 255 . These may be represented as either decimal or hexadecimal numbers.

2. RGB to Grayscale Conversion Steps / Algorithm i. Traverse through entire input image array.

ii. Read individual pixel color value (24-bit).

iii. Split the color value into individual $R, G$ and $\mathrm{B} 8$ bit values.

iv. Calculate the grayscale component (8bit) for given $R, G$ and $B$ pixels using a conversion formula.

v. Compose a 24-bit pixel value from 8-bit grayscale value.

vi. Store the new value at same location in output image.

3. Thresholding Steps / Algorithm

i. Traverse through entire input image array.

ii. Read individual pixel color value (24-bit) and convert it into grayscale.

iii. Calculate the binary output pixel value (black or white) based on current threshold.

iv. Store the new value at same location in output image.

\section{COLOR - $\quad$ GRAYSCALE - $\quad$ BLACK \& WHITE}
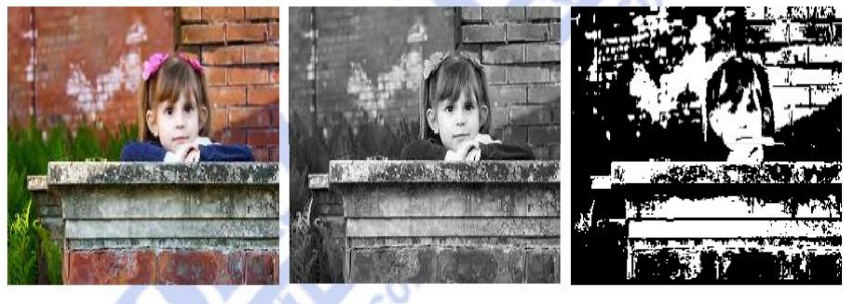

FIG. 2 CONVERSION OF COLOR IMAGE TO GRAYSCALE TO BLACK \& WHITE

\section{EXPERIMENTAL RESULTS}

In this section, some experimental are performed to show the performance of the proposed scheme. Besides, an implemented HMI system also is presented in this section to show an application example of gesture control. In this experiment, the video input device is a common webcam. In the image preprocessing, the proposed scheme uses the noise filter and equalization method to enhance the important information for the hand detection and gesture recognition. The results of the Gaussian filter and the median filter and these noises filters both can reduce most noises and still can remain feature information for hand detection and gesture recognition. 


\section{CONCLUSION}

The gesture control technique has become a new developmental trend for many human-based electronics products. The human-machine interface for users to control some service system just by their hands. The proposed scheme can be further applied to be HMI for other applications, such as, intelligent televisions, playing games, robots, bulletin board and so on.

\section{ACKNOWLEDGMENT}

The Apart from our own, the success of this report depends largely on the encouragement and guidelines of many others. We are especially grateful to our guide Prof. Bhavana S. Pansare who has provided guidance, expertise and encouragement. We express our heartfelt gratefulness to Prof. S. B. Ingle, Head of Computer Engineering Department, NMIET, for their stimulating supervision whenever required during my seminar work. We would like to put forward my heartfelt acknowledgement to all our classmates, friends and all those who have directly or indirectly provided their overwhelming support during our seminar work and the development of this report.

\section{REFERENCES}

[1] Y. Wu, T. Huang, Vision-based gesture recognition: a review, in gesture-based communications in HCI, Lecture Notes in Computer Science, Vol. 1739, Springer, Berlin, pp. 103-115, 1999.

[2] C. Pickering, K. Burnham, and M. Richardson, A research study of hand gesture recognition Technologies and Applications for Human Vehicle Interaction, in Proc. of the 3rd Institution of Engineering and Technology Conference on Automotive Electronics, pp. 1-15, 2007.

[3] W. Wierwille, Visual and manual demands of in car controls and displays. In Automotive Ergonomics, Ed. by Peacock, B.Karwowski, B., Taylor and Francis, pp. 299-313, 1993.

[4] C. Pickering, The search for a safer driver interface: a review of gesture recognition human machine interface, Computing \& Control Engineering Journal, Vol. 16, pp. 3440, 2005.

[5] L., Wang, W. Hu, and T. Tan, Recent developments in human motion analysis, Pattern Recognition, Vol. 36, pp. 585-601, 2003.

[6] K. Imagawa, S. Lu, and S. Igi, Color-based hands tracking system for sign language recognition, in Proc. of the Third
IEEE International Conference on Automatic Face and Gesture Recognition, pp. 462-467, 1998.

[7] L. Bretzner, I. Laptev1, and T. Lindeberg, Hand gesture recognition using multi-scale colour features, Hierarchical Models and Particle Filtering, in Proc. of the Fifth IEEE International Conference on Automatic Face and Gesture Recognition, pp. 423, 2002.

[8] A. Yilmaz, and M. Shah, Actions as objects: a novel action representation, in Proc. of the 2005 IEEE Computer Society Conference on Computer Vision and Pattern Recognition, 2005

[9] A. El-Sawah, N. D. Georganas, and E. M. Petriu, A Prototype for 3D Hand Tracking and Posture Estimation, IEEE Transactions on Instrumentation and Measurement, Vol. 57, pp. 1627-1636, 2008.

[10] W. Lu, and J. Little, Tracking and recognizing actions at a distance, in Proc. of the ECCV Workshop on Computer Vision Based Analysis in Sport Environments, pp. 49-60, 2006

[11] W. James and J. Little, Simultaneous tracking and action recognition using the PCA-HOG descriptor, in Proc. of the Third Canadian Conference on Computer and Robot Vision, pp. 6, 2006.

[12] L. Gorelick, M. Blank, E. Shechtman, M. Irani, and R. Basri, Actions as space-time shapes, IEEE Trans. Pattern Analysis and Machine Intelligence Vol. 29, No. 12, pp. 2247-2253, 2007.

[13] S. Calderara, R. Cucchiara, and A. Prati, Action signature: A novel holistic representation for action recognition, in Proc. of theInternational Conference on Advanced Video and Signal Based Surveillance, IEEE Computer Society Press, Washington, pp. 121128, 2008.

[14] C. Schuldt, I. Laptev, and B. Caputo, Recognizing human actions: a local svm approach, in Proc. of the International Conference on Pattern Recognition, Vol. 3, IEEE Computer Society Press, Cambridge, UK, pp. 32-36, 2004.

[15] P. Scovanner, S. Ali., and M. Shah, A 3-dimensional sift descriptorand its application to action recognition, in Proc. of the $15^{\text {th }}$ international conference on Multimedia, ACM, pp. 357-360, 2007.

[16] J. Liu, and M. Shah, Learning human actions via information maximization, in Proc. of the International Conference on Computer Vision and Pattern Recognition, IEEE Computer Society Press, Anchorage, Alaska, pp. 1-8, 2008

[17] Q. Luo, X. Kong, G. Zeng, and J. Fan, Human action detection via boosted local motion histograms, Machine Vision and Applications, Vol. 21, pp. 377-389, 2010.

[18] N. Otsu, A threshold selection method from gray-level histograms, IEEE Trans. Sys., Man., Cyber. Vol. 9, pp. 6266, 1979.

[19] M. B. Dillencourt and H. Samet and M. Tamminen, A general approach to connected-component labeling for arbitrary image representations, Journal of the ACM, Vol. 39, pp. 253-280, 1992. 